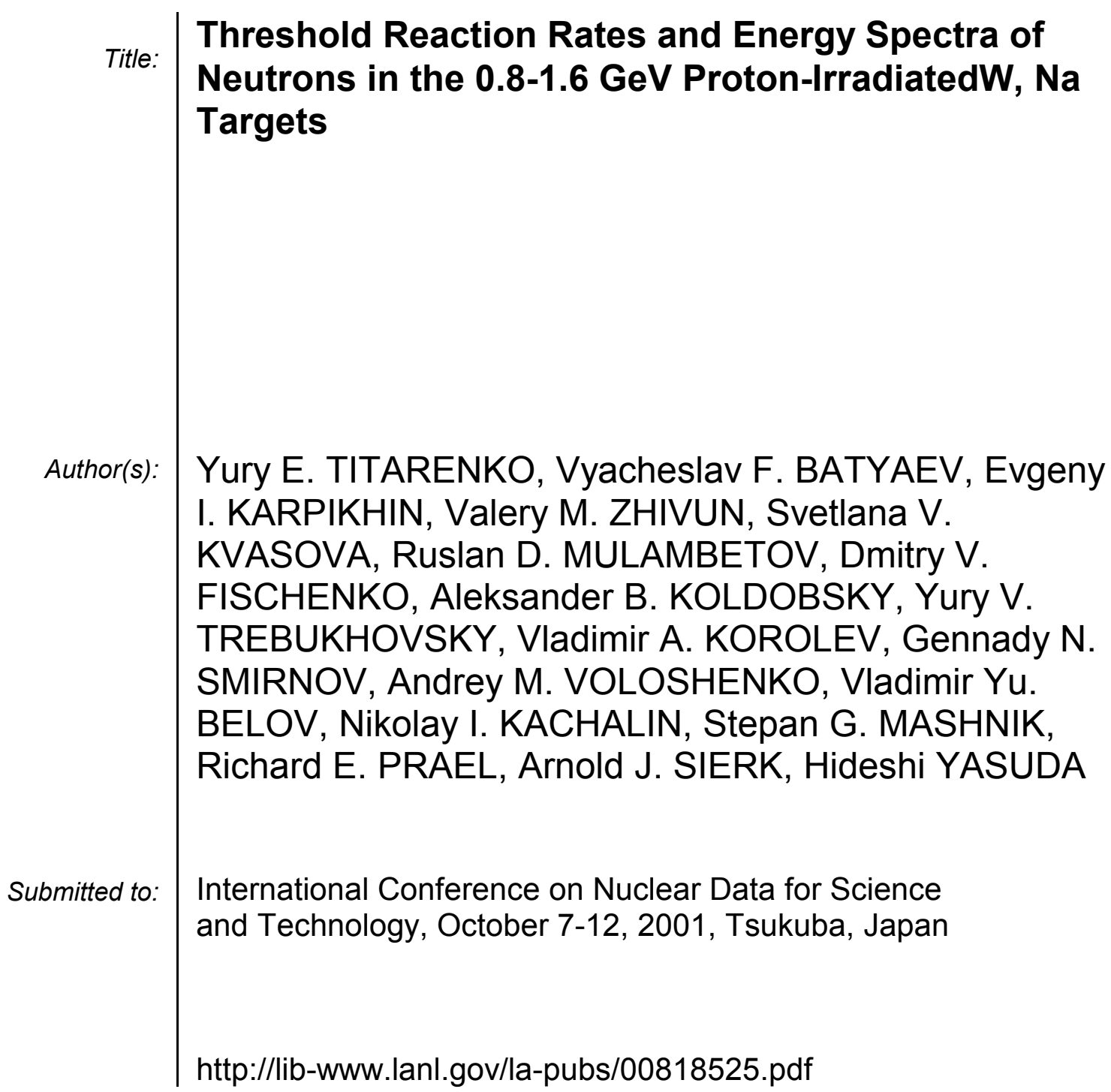




\title{
Threshold Reaction Rates and Energy Spectra of Neutrons in the 0.8-1.6 GeV Proton-Irradiated W, Na Targets
}

\author{
Yury E. TITARENKO ${ }^{1, *}$, Vyacheslav F. BATYAEV ${ }^{1}$, Evgeny I. KARPIKHIN ${ }^{1}$, Valery M. ZHIVUN ${ }^{1}$, Svetlana V. KVASOVA ${ }^{1}$, \\ Ruslan D. MULAMBETOV ${ }^{1}$, Dmitry V. FISCHENKO ${ }^{1}$, Aleksander B. KOLDOBSKY ${ }^{1}$, Yury V. TREBUKHOVSKY ${ }^{1}$, \\ Vladimir A. KOROLEV ${ }^{1}$, Gennady N. SMIRNOV ${ }^{1}$, Andrey M. VOLOSHENKO ${ }^{2}$, Vladimir Yu. BELOV ${ }^{3}$, \\ Nikolay I. KACHALIN ${ }^{3}$, Stepan G. MASHNIK ${ }^{4}$, Richard E. PRAEL ${ }^{4}$, Arnold J. SIERK ${ }^{4}$, Hideshi YASUDA ${ }^{5}$ \\ ${ }^{1}$ Institute for Theoretical and Experimental Physics, B.Cheremushkinskaya 25, 117259 Moscow, Russia \\ ${ }^{2}$ Keldysh Institute of Applied Mathematics, Miusskaya Sq. 4, 125047 Moscow, Russia \\ ${ }^{3}$ Russian Federal Nuclear Center - VNIIEF, 607190, Sarov (Arzamas-16), Nizhny Novgorod region, Russia \\ ${ }^{4}$ Los Alamos National Laboratory, Los Alamos, NM 87545, USA \\ ${ }^{5}$ Japan Atomic Energy Research Institute, Tokai-mura, Ibaraki 319-1195, Japan
}

\begin{abstract}
Considering the prospects of using the W-Na target assemblies in ADS facilities, the experiments were made to study the nuclear-physics characteristics of $\mathrm{W}$ and $\mathrm{Na}$, and the composite structures thereof in their interactions with $0.8-\mathrm{GeV}$ and $1.6-\mathrm{GeV}$ protons. The neutron and proton-induced reaction rates were measured inside, and on the surface of, a cylinder-shaped heterogeneous W-Na assembly together with the double-differential spectra of secondary neutrons emitted from different-depth $\mathrm{W}$ and Na discs. The measurement results were simulated by the LAHET, CEM2k, and KASKAD-S codes in terms of the latest versions of nuclear databases.
\end{abstract}

\section{KEYWORDS: proton irradiation, neutron spectra, reaction rates, spallation, simulation codes.}

\section{Introduction}

Tungsten is regarded as belonging to the most promising class of target materials in the present-day conceptual designs of ADS facilities. Tungsten is sufficiently easy to handle, is of a high density, shows the desired set of nuclear-physics characteristics, and does not exhibit chemical reactivity and biological toxicity (contrary to $\mathrm{Pb}$ and $\mathrm{Hg}$ ).

At the same time, the actual target designs include not only the neutron sources made of heavy materials, but also heat exchangers with coolants. Na is often proposed to be a coolant, considering that the Na-based technologies have been studied quite properly. Besides, Na can also be used in the heterogeneous target designs to level the energy deposit within the target volume.

In view of the above, accumulation and estimation of the microscopic and group nuclear constants for the said materials and for the assemblies thereof have become urgent. Bearing in mind that the target structures are very complicated and can be subject to ample alterations, the computational methods based on the latest simulation codes and nuclear databases can only be used for that purpose. On the other hand, as mentioned repeatedly in the relevant publications (see ${ }^{1)}$, for instance), the present-day codes are often devoid of the predictive power required by the actual ADS facility designs.

A necessity has arisen, therefore, for the simulation codes to be tested by the results of experimenting with the prototypes of the target parts and units. It should not be forgotten either that the said experimental results can, and certainly must, be used as independent nuclear constants. The present work describes two groups of the experiments, namely,

- studying the threshold reaction rates inside, and on the surface of, a $0.8 \mathrm{GeV}$ proton-irradiated lamellate heterogeneous W-Na target;

\footnotetext{
${ }^{*}$ Corresponding author, Tel. +7-095-123-6383, Fax. +7- 095-127 -0543, E-mail: Yury.Titarenko@itep.ru
}

- measuring the double-differential neutron spectra from $0.8 \mathrm{GeV}$ and $1.6 \mathrm{GeV}$ proton-irradiated $\mathrm{W}$ and $\mathrm{Na}$ targets.

The experimental results were simulated by the LAHET code (in both cases), by the KASKAD-S code (the threshold reaction rates), and by the CEM2k code (the neutron spectra).

\section{Experiment}

The experiments were made with the ITEP U-10 proton synchrotron beams of $7.2 \cdot 10^{10} \mathrm{p} /$ pulse (the reaction rates) and $\sim 1 \cdot 10^{5} \mathrm{p} /$ pulse (the secondary neutron spectra) intensities.

The general experimental design of measuring the reaction rates has been described $\mathrm{in}^{2)}$. The measurements were made by the gamma-spectrometry method using 142 experimental samples of thirteen single-isotope and high isotopic enriched materials $\left({ }^{12} \mathrm{C},{ }^{19} \mathrm{~F},{ }^{27} \mathrm{Al},{ }^{59} \mathrm{Co},{ }^{63} \mathrm{Cu},{ }^{65} \mathrm{Cu},{ }^{64} \mathrm{Zn}\right.$, ${ }^{93} \mathrm{Nb},{ }^{115} \mathrm{In},{ }^{169} \mathrm{Tm},{ }^{181} \mathrm{Ta},{ }^{197} \mathrm{Au}$, and ${ }^{209} \mathrm{Bi}$ ). The 0.03-2.1 $\mathrm{g} / \mathrm{cm}^{2}$ disc-shaped samples were placed at 84 points inside, and on the surface of, a heterogeneous W-Na assembly. The continuous irradiation time was 10 hours, with a $6.5 \cdot 10^{14}$ integral proton fluence. After the irradiation, the samples were gamma-spectrometered using the GC2518 Ge detector of a $1.8 \mathrm{keV}$ resolution in the $1332 \mathrm{keV}$ gamma-line. The resultant gamma-spectra were processed by the GENIE2000 code. The secondary nuclide were identified by the ITEP-developed SIGMA code using the PCNUDAT database.

The double-differential spectra of secondary neutrons were measured by the techniques ${ }^{3)}$ using a TOF spectrometer. The neutrons were recorded with the BICRON 5MAB1F D12.7* $15.2 \mathrm{~cm}$ detectors based on BC501 liquid scintillator. The projectile proton energies were $0.8 \mathrm{GeV}$ and 1.6 $\mathrm{GeV}$; the neutrons were detected at $30^{\circ}, 60^{\circ}, 90^{\circ}, 120^{\circ}$, and $150^{\circ}$. The gamma-background was discriminated by the pulse shape. The absolute neutron detection efficiency was calculated by the SCINFUL ${ }^{4)}$ and $\mathrm{CECIL}^{5)}$ codes. 


\section{Computational simulation and comparison with experimental data}

The experimental data were simulated by the LSC code system $^{6}$ ) using the LAHET code (in both cases), the KASKAD-S code ${ }^{7)}$ (threshold reaction rates), and the CEM2k code $^{8)}$ (neutron spectra). The simulation techniques are described in detail $\mathrm{in}^{2)}$.

The results of comparing between the experimental and simulated data are presented in Figs. 1-3 (reaction rates) and 4-5 (secondary neutron spectra). The mean squared deviation factor $\langle\mathrm{F}\rangle$ was used as a criterion for agreement between the calculated and experimental reaction rates.

Beside the comparison between the experimental and simulated results of the present work, Fig. 5 shows the results of work $^{9)}$, which is the only work that admits a straightforward comparison with our data.

\section{Conclusions}

The experimental data and the results of testing the simulation codes have permitted the following conclusions:

- many of the measured reactions cannot be reproduced by simulation in terms of the present-day cross section libraries (MENDL2 and MENDL2P) because of their restricted energy range ${ }^{10)}\left(\mathrm{E}_{n}<100 \mathrm{MeV}, \mathrm{E}_{p}<200 \mathrm{MeV}\right)$;

- the results obtained indicate that the cumulating effect in the high-energy neutron reactions has to be allowed for;

- the studied reaction rates inside, and on the surface of, a heterogeneous W-Na target indicate that the codes based on Monte-Carlo techniques (e.g., LAHET) are much more promising compared with the codes based on the discrete-ordinate algorithm (e.g., KASKAD-S);

- theoretical models for separating the secondary product yields between the isomeric states have urgently to be constructed with a view to updating the simulation codes; the absence of such a model prevents from a reliable simulation-to-experimental comparison for a number of reactions $\left({ }^{115} \operatorname{In}(\mathrm{n}, \mathrm{p})^{115 g} \mathrm{Cd}\right.$, for instance);

- the quality of the calculated reproduction of the doubledifferential spectra of secondary neutrons is much higher in the case of $\mathrm{W}$ compared with $\mathrm{Na}$, the fact that can qualitatively be explained by the drawbacks of the presentday theoretical models for reactions with small-mass nuclei at intermediate and high energies;

- additional measurements of the space-energy characteristics of secondary neutrons seems to be urgent because the available data are scanty and preclude any final conclusion concerning the reasons for the discrepancies in the experimental results of the given type; moreover, a nuclear database that could have been safely used in practice is impossible to compile using the present-day experimental data.

\section{Acknowledgment}

The authors are indebted to Dr. S. Meigo (JAERI) for his assistance in calculating the detection efficiency, to Dr. V. L. Romodanov (MEPI, Moscow) for his assistance in calibrating the detectors, to Drs. V. N. Kostromin and I. A. Vorontsov (ITEP, Moscow) for their assistance in carrying out the experiments, and to Dr. F. E. Chukreev (KIAE, Moscow) for discussion of the results.

The work has been carried out under the ISTC Project \#1145 supported by JAERI (Japan). In part, the work has been supported by the U.S. Department of Energy.

\section{References}

1) Yu. E. Titarenko et al., "Experimental and Theoretical Study of the Residual Product Nuclide yields in Thin Targets Irradiated with 100-2600 MeV Protons," this proceedings.

2) Yu. E. Titarenko et al., "Threshold Reaction Rates Inside and on the Surface of Thick W-Na Target Irradiated with $0.8 \mathrm{GeV}$ Protons," in Proc. of Int. Workshop on Shielding Aspects of Accelerators, Irradiation and Target Facilities (SATIF-5), OECD Headquarters, Paris, France, July 20-21, 2000, pp. 29-36; "Study of Threshold Reaction Rates Inside and on the Surface of a $0.8-\mathrm{GeV}$ Proton-Irradiated Thick W-Na Target," in Proc. of AccApp01/ADTTA'01 Nucl. App. in the New Millennium Int. Meet., Reno, NV, Nov. 11-15, 2001; LA-UR-01-4788.

3) Yu. E. Titarenko et al., "Spectral Measurements of Neutrons from $\mathrm{Pb}, \mathrm{W}$, And $\mathrm{Na}$ Targets Irradiated by 0.8 and $1.6 \mathrm{GeV}$ Protons," in Proc. of Int. Workshop on Shielding Aspects of Accelerators, Irradiation and Target Facilities (SATIF-5), OECD Headquarters, Paris, France, July 20-21, 2000, pp. 37-46; Yu. E. Titarenko et al., "Measurements of Neutron Spectra in 0.8GeV and 1.6-GeV Proton-Irradiated W and Na Thick Targets," in Proc. of AccApp01/ADTTA'01 Nucl. App. in the New Millennium Int. Meet., Reno, NV, Nov. 11-15, 2001; LA-UR-01-4789.

4) J. K. Dickens, ORNL-6452, (1988); v. 4, S. Meigo, priv. comm.

5) R. A. Cecil et al., Nucl. Instrum. Methods, 161, 439 (1979); v. KSU, S. Meigo, priv. comm.

6) R. E. Prael, H. Lichtenstein, LANL Report LA-UR-89-3014 (1989).

7) A. M. Voloschenko, "An Algorithm for Spallation Target Neutronics and Shielding Calculations," in Proc. of International Conference on Mathematics and Computations, Reactor Physics, and Environmental Analyses in Nuclear Applications, 27-30 September, 1999, Madrid, Spain, Vol. 2, p. 975; A. M. Voloschenko et al., "Using Discrete Ordinate Method in the Spallation Target Neutronics and Shielding Calculations," in Proc. 3-rd International Conference on Accelerator Driven Transmutation Technologies and Applications, June 7-11, 1999, Pruhonice, Chech Republic, Paper Code We-O-E12.

8) S. G. Mashnik and A. J. Sierk, "CEM2k - Recent Developments in CEM," in Proc. of the Fourth International Topical Meeting on Nuclear Applications of Accelerator Technology (AccApp00), November 13-15, 2000, Washington, DC, USA, (American Nuclear Society, La Grange Park, IL, USA, 2001) pp. 328-341; http://xxx.lanl.gov/ps/nucl-th/001164.

9) W. B. Amian et al., Nucl. Sci. Eng., 112, 78 (1992); W. B. Amian et al., Nucl. Sci. Eng., 115, 1 (1993).

10) Yu. N. Shubin et al., IAEA Report INDC(CCP)-385, 1995. 


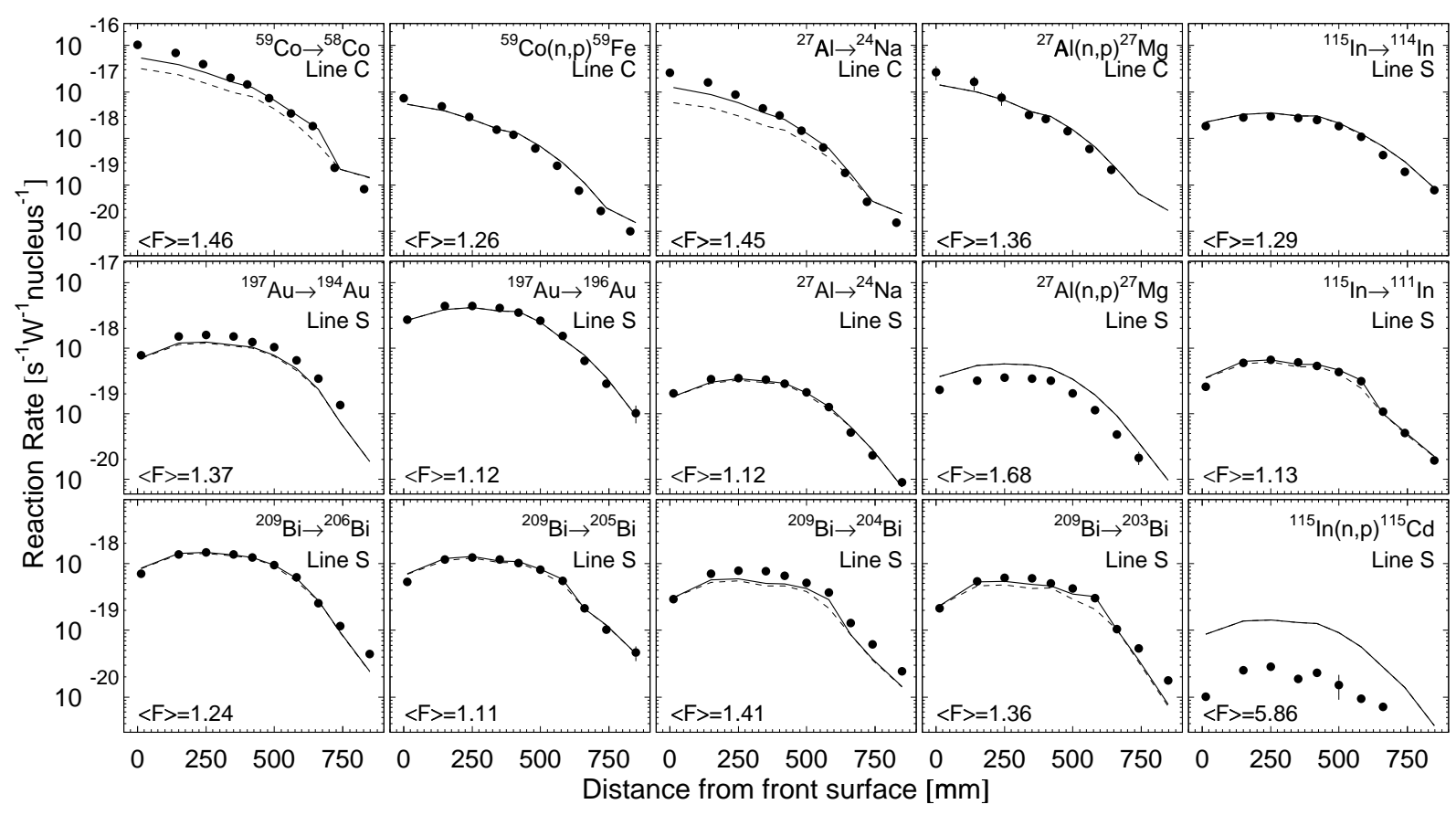

Fig. 1 The experimental and the LCS calculated reaction rates. The dashed line is the neutron contribution to the reaction rates. The mean squared deviation factor $\langle\mathrm{F}\rangle$ is also shown. The presented reaction rates have been normalized to the proton beam power.

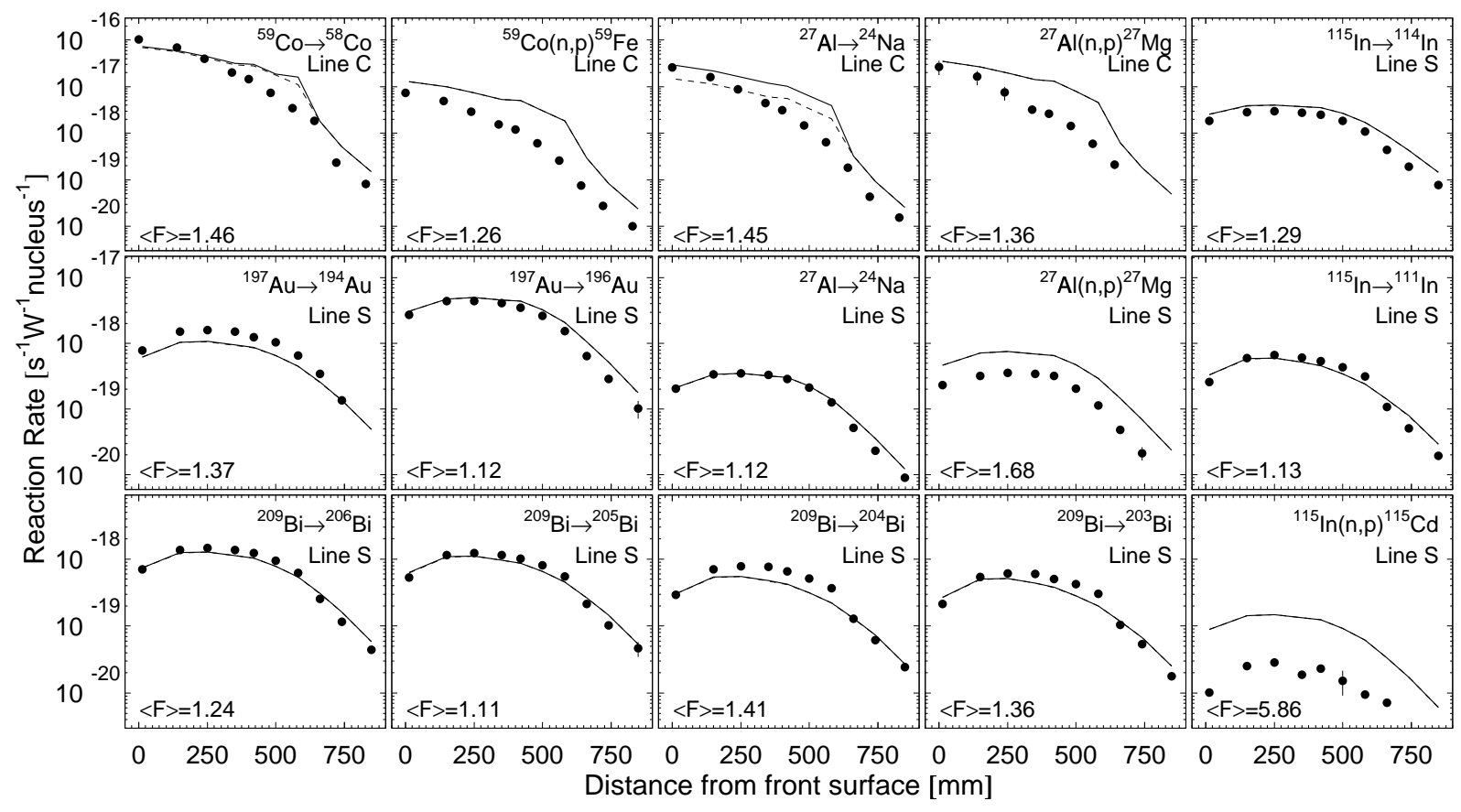

Fig. 2 The experimental and the KASKAD-S calculated reaction rates. The dashed line is the neutron contribution to the reaction rates. The mean squared deviation factor $\angle \mathrm{F}>$ is also shown. The presented reaction rates have been normalized to the proton beam power.
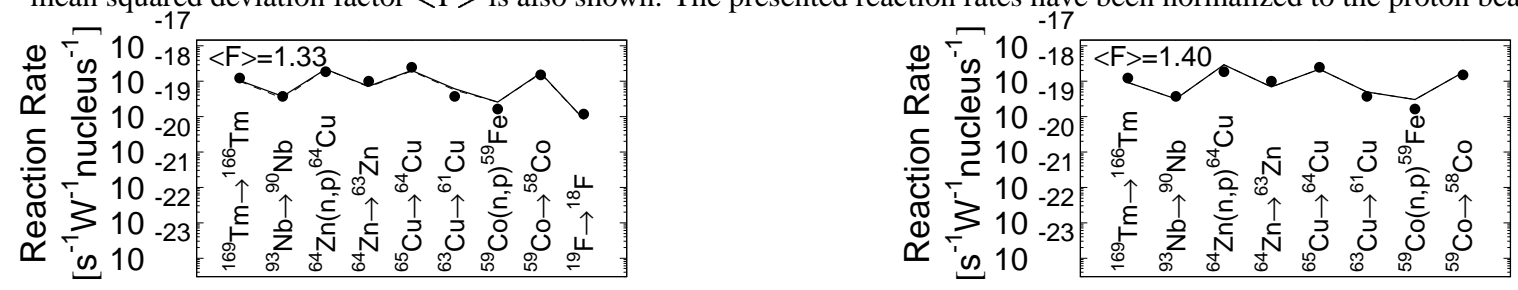

Fig. 3 The experimental and the LCS (left figure) and KASKAD-S (right figure) calculated reaction rates at the surface of second W-disk. The dashed line is the neutron contribution to the reaction rates. The mean squared deviation factor $<\mathrm{F}>$ is also shown. The presented reaction rates have been normalized to the proton beam power. 


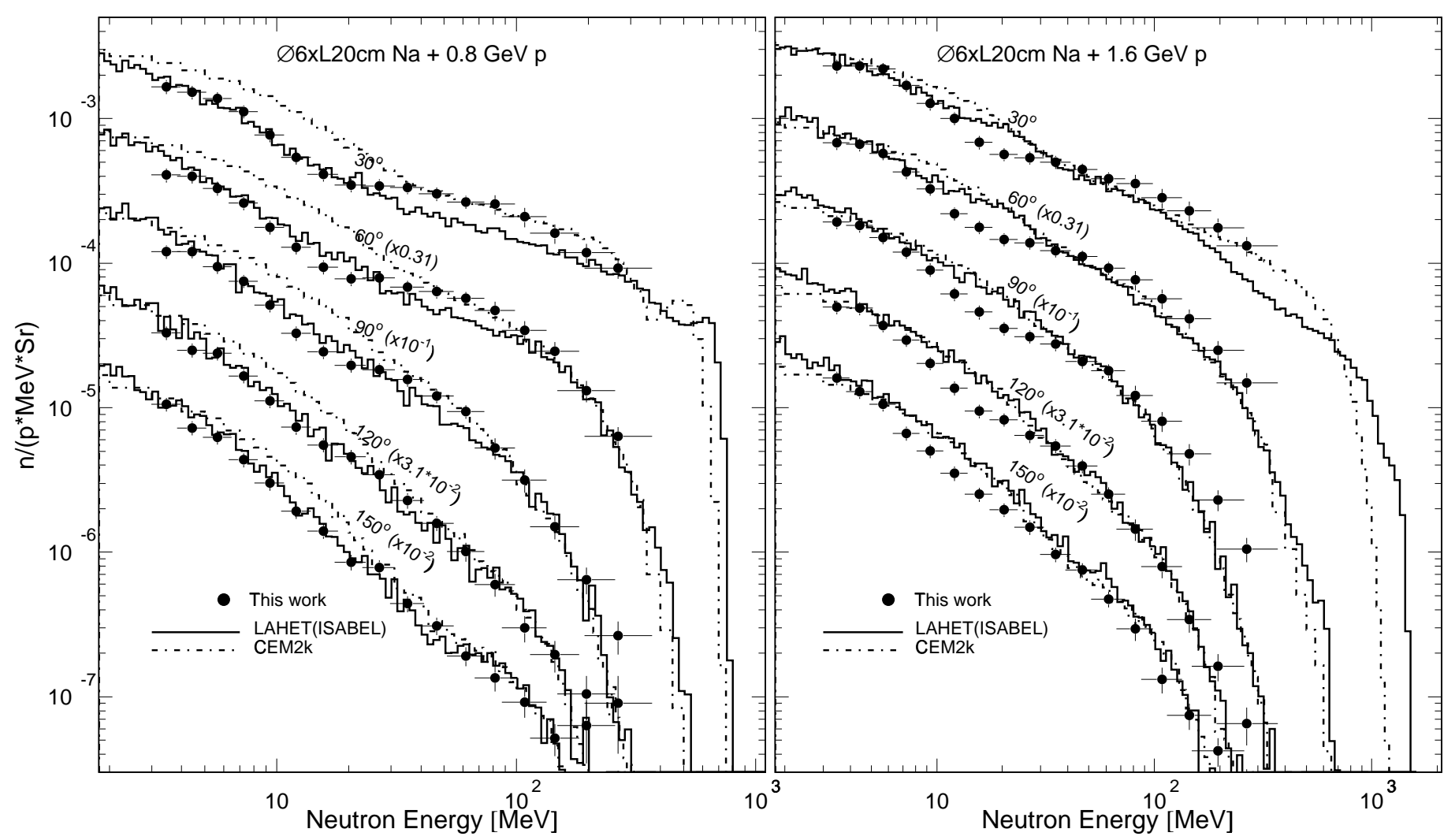

Fig. 4 The double differential neutron spectra measured in the present work from D6xL20 cm Na irradiated by the 0.8 (the left-hand panel) and 1.6 (the right-hand panel) $\mathrm{GeV}$ protons, together with the $\mathrm{LAHET}^{6)}$ and CEM2k ${ }^{8)}$ code calculation results.

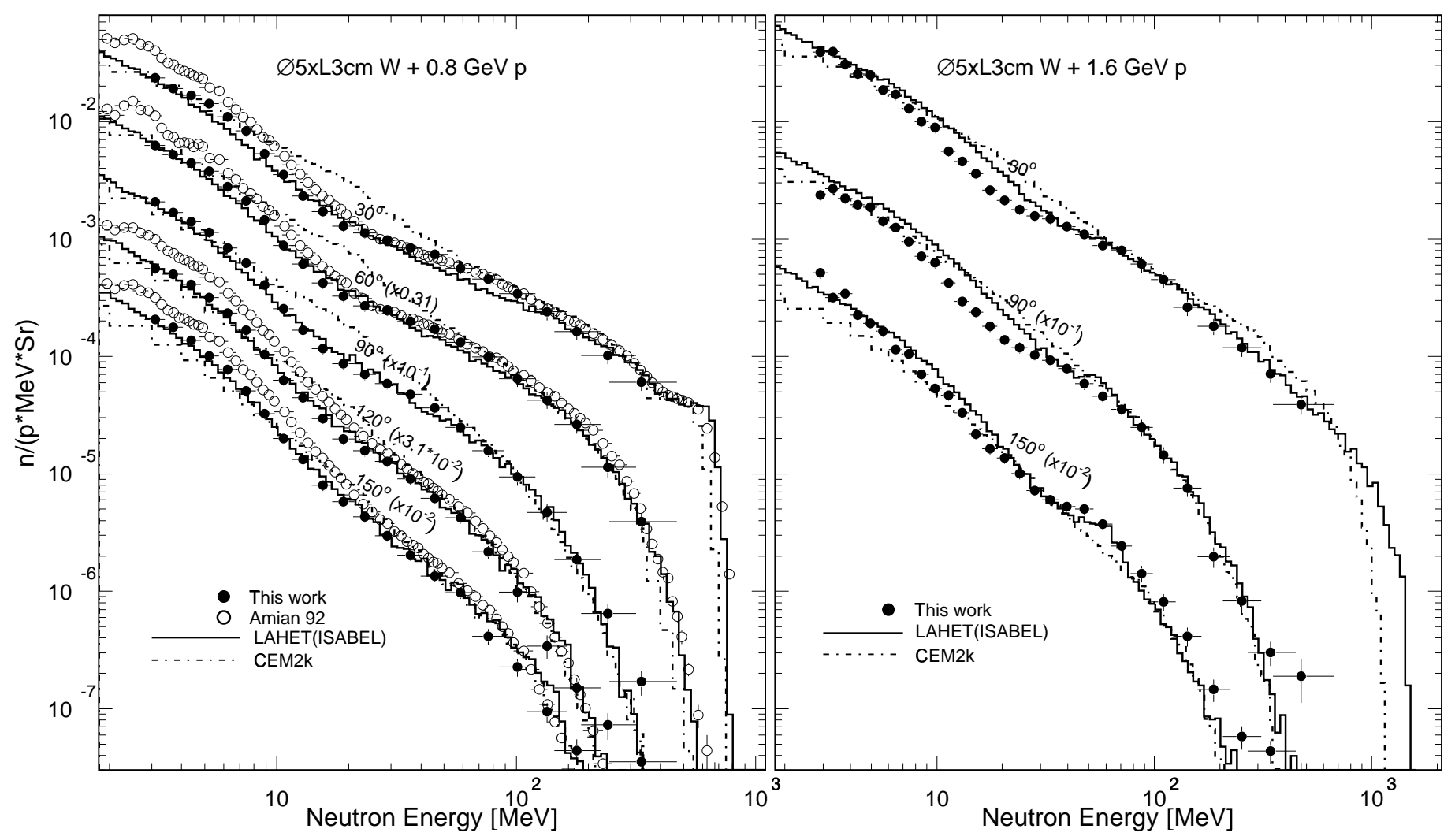

Fig. 5 The double differential neutron spectra from D5xL2.935 cm W irradiated by the $0.8 \mathrm{GeV}$ (the left-hand panel) and $1.6 \mathrm{GeV}$ (the right-hand panel) protons, as measured in the present work and in ${ }^{9)}$ for $0.8 \mathrm{GeV}$ protons, together with the $\mathrm{LAHET}^{6)}$ and $\mathrm{CEM}^{\mathrm{k}} \mathrm{k}^{8)}$ code calculation results. 